\title{
The role of baicalin on carbon tetrachloride induced liver fibrosis
}

\author{
Mengting LI*; YI-ER QIU; KaIFENG ZHENG \\ Department of Gastroenterology, the Affiliated People's Hospital of Ningbo University, Ningbo, 315040, China
}

Key words: Liver Fibrosis, Baicalin, Cell Apoptosis

\begin{abstract}
The effect of the baicalin, a bio-active flavonoid extracted from Scutellaria baicalensis Georgi, on the carbon tetrachloride $\left(\mathrm{CCl}_{4}\right)$ induced liver fibrosis was investigated. To compare the effect of baicalin on the liver fibrosis, five different groups of rats treated by 100,200 , and $400 \mathrm{mg} / \mathrm{kg}$ baicalin were studied. Upon $\mathrm{CCl}_{4}$ treatment, the levels of procollagen type III, aspartate aminotransferase, aminotransferase, hyaluronic acid, and hydroxyproline were significantly increased, whereas the superoxide dismutase and glutathione peroxidase content were decreased. These changes in the biochemical parameters, which are associated with liver function, were significantly attenuated by the baicalin treatment, suggesting that baicalin can suppress the liver fibrosis induced by $\mathrm{CCl}_{4}$. Moreover, the histological staining analysis demonstrated that baicalin could effectively inhibit the degree of liver cell injury. The protein expression of $\mathrm{AKT} / \mathrm{JAK} 2 / \mathrm{ERK}$ in the serum were markedly increased by $\mathrm{CCl}_{4}$ but suppressed by the treatment of baicalin in a dose-dependent manner, implying that baicalin can attenuated cell apoptosis induced by $\mathrm{CCl}_{4}$. Overall, these results suggest that baicalin effectively protects hepatocytes from the $\mathrm{CCl}_{4}$ oxidative damage, likely due to the inhibition of free radical generation and cell apoptosis during the liver injury.
\end{abstract}

\section{Introduction}

Liver disease is one of the major global challenges. According to the Office for National Statistics in the United Kingdom, liver disease is the fifth most common cause (Toledano et al., 2019). Liver fibrosis is a common chronic liver disease, and it is considered as a critical issue due to its high rate of morbidity and mortality (Huang et al., 2014; Sun et al., 2007). Hepatitis viral infection is the most common cause. Drug abuse, autoimmune disorders, metabolic disorders, and biliary obstruction due to mineral overload are attributed to the liver fibrosis (Friedman, 2003; Kisseleva and Brenner, 2006). Many environmental toxins can also cause liver injury and fibrosis, which is often characterized as the accumulation of extracellular matrix protein and collagen in the perisinusoidal space (Dai et al., 2009; Sun et al., 2012). Moreover, it was found that serious liver fibrosis leads to cirrhosis and liver failure eventually (Are et al., 2020; Zimmermann et al., 2019). However, treatment of liver diseases still remains as a therapeutic challenge. Therefore, there is a need to develop effective therapeutic strategies that will decrease the extent of liver fibrosis or enhance the liver regeneration. Despite technology advances in medicine, only few medical treatment approaches are available for the liver fibrosis (Sun et al., 2012).

*Address correspondence to: Mengting Li, limengting1992@foxmail.com Received: 07 August 2020; Accepted: 25 September 2020
Meanwhile, modern medicine lacks reliable and effective liver protective activities. Therefore, there has been considerable interest in the development of alternative and complementary medicine for the treatment of liver diseases.

Although the pathogenesis of liver fibrosis is quite complicate, the understanding of biochemical and cellular factors associated with liver fibrosis is gradually increasing. Several reports have suggested that oxidative stress can play a key role in the liver fibrosis (Cederbaum et al., 2009; Poli, 2000). For instance, free radicals, as found during the oxidative process in cells, can attack unsaturated fatty acids of cell membranes, resulting in cell peroxidation, destructing DNA and protein (Sastre et al., 2007). These processes eventually cause various liver injuries. It has been shown that traditional herb medicine, which contains antioxidants, can be an effective approach to prevent liver fibrogenesis (Gebhardt, 2002; Lin et al., 2018).

For example, the radix of Scutellaria baicalensis is an eastern traditional medicine, and it has been used as a relaxant for smooth muscle and an anti-inflammatory reagent (Huang et al., 2018; Liu et al., 2019). It has also been used as an ingredient of herb mixture for the hepatoprotective agent (Dong et al., 2020; Gao et al., 1995; Huang et al., 2012; Liu et al., 2015a; Liu et al., 2015b; Wu et al., 2018; Yin et al., 2018; Yu et al., 2020; Zhou et al., 2018). Baicalin is the major active component of the isolated root of Scutellaria baicalensis, and it acts as a free radical scavenger for reactive oxygen species and has an 
anti-inflammatory activity (Gao et al., 1995; Zhou et al., 2018). It was found that the baicalin can inhibit the $\mathrm{H}_{2} \mathrm{O}_{2}$-induced liver injury caused by suppression of oxidation in the cell ( $\mathrm{Yu}$ et al., 2020). It can also inhibit the activation of redox sensitive nuclear factor- $\kappa \mathrm{B}(\mathrm{NF}-\kappa \mathrm{B})$ in kidney from the old rats (Liu et al., 2015a; Yin et al., 2018). However, information about the hepatoprotective effect of baicalin is very limited.

Carbon tetrachloride $\left(\mathrm{CCl}_{4}\right)$ is commonly employed as an inducer of the liver injury in small animals (Huang et al., 2014; Yu et al., 2020). It is known that $\mathrm{CCl}_{4}$ is hepatotoxic as well as nephrotoxic to humans (Kodavanti et al., 1989; Ritesh et al., 2015). It has been reported that hepatic necrosis induced by $\mathrm{CCl}_{4}$ involves the activation by a microsomal cytochrome P450-depentent (Sun et al., 2007) monooxygenase system, resulting in the generation of ROS and trichloromethyl free radicals. It eventually leads to damage of cell membrane (Mccay et al., 1984), following by the release of inflammatory mediators from the activated macrophages. They are believed to be the cause of the $\mathrm{CCl}_{4}$ induced hepatic injury. In this work, we investigated the role of baicalin in $\mathrm{CCl}_{4}$ induced liver injury and how it can protect the liver injury. The possible molecular mechanism and the effect of inhibition of the oxidative stress and inflammation will be discussed.

\section{Materials and Methods}

\section{Materials}

Carbon tetrachloride $\left(\mathrm{CCl}_{4}\right)$ was purchased from Aladdin (part no.: C112043, China). Commercial kits for SOD, GSHPx, hydroxyproli, MDA, AST/GOT, and ALT/GPT were purchased from Jiancheng Co. (Nanjing, China). 85\% Baicalin was purchased from Fusion Biology Co. (Part No. 21967-41-9, China).

Experiments were carried out on 17 ICR male rats (Jestier, China) of 18-22 g body weight. They were housed at $22-24^{\circ} \mathrm{C}$ and exposed to alternate cycles of $12 \mathrm{~h}$ light and dark. They were given free access to a standard pellet diet and tap water. The animal study was approved by the Laboratory Animal's Ethic Committee of the Hospital. It was carried out throughout the experiment following the international laboratory animal use and care guideline.

\section{Experimental model and treatment}

We first prepared a $2 \% \mathrm{CCl}_{4}$ solution by mixing $\mathrm{CCl}_{4}$ and corn oil thoroughly mixed for $2 \mathrm{~min}$. Then the $\mathrm{CCl}_{4}$-corn oil solution was administered subcutaneously at a dose of $0.1 \mathrm{~mL} / \mathrm{kg}$ of body weight, twice per week and continued for 6 weeks, in order to induce the liver fibrosis.

The animals were randomly divided into 5 groups: (1) Control group (normal rats), in which 3 rats were injected subcutaneously with corn oil with no $\mathrm{CCl}_{4}$. (2) $\mathrm{CCl}_{4}$ control group, in which 5 rats were administered $2 \% \mathrm{CCl}_{4}$ without adding baicalin. (3) $\mathrm{CCl}_{4}+$ low dose baicalin group, in which 3 rats were administered with $2 \% \mathrm{CCl}_{4}$ and baicalin $\left(100 \mathrm{mg} / \mathrm{kg}\right.$ ). (4) $\mathrm{CCl}_{4}+$ intermediate dose baicalin group, in which 3 rats were administered with $2 \% \mathrm{CCl}_{4}$ and baicalin mixture $(200 \mathrm{mg} / \mathrm{kg})$ (5) $\mathrm{CCl}_{4}+$ high dose baicalin group, in which 3 rats were administered with $2 \% \mathrm{CCl}_{4}$ and baicalin $(400 \mathrm{mg} / \mathrm{kg})$.
At the end of six weeks, animals were scarified. The blood samples were obtained from orbital veins. The serum samples were collected by centrifugation in a Sorvall RC centrifuge (Thermo Scientific, Germany) of the whole blood at 4000 $\mathrm{rpm}$ for $10 \mathrm{~min}$. The samples were stored frozen at $-80^{\circ} \mathrm{C}$ until further use. The livers were immediately removed and were fixed in $10 \%$ formalin for histological analysis.

Liver index, kidney index, and spleen index

Relative weights of liver, kidney and spleen were represented as the percentage of the total body weight in gram.

\section{Biochemical metabolic parameters}

The activities of ALT and AST were measured by spectrophotometry using commercial kits, according to the manufacturer's instructions. The levels of LN, HA, PC III were obtained by radio-immunoassay kits, according to the manufacturer's instructions. TGF-betal was determined by the rat ELISA kit.

\section{Evaluation of oxidative stress and antioxidant status}

The tissue homogenates of livers were used to determine SOD, MDS, and GDH-PX levels, according to the manufacturer's instructions.

\section{Evaluation of collage}

Liver collage concentration was determined by measuring the level of hydroxyproline, according to the method published previously (Sun et al., 2007).

\section{Histological observation}

Livers were isolated from rats and the tissues were fixed using $10 \%$ formalin for $24 \mathrm{~h}$. The fixed tissues were then dehydrated and immersed in paraffin solution, followed by cutting into small sections for staining. The hematoxylin-eosin staining was used to observe liver injury. The tissues were stained with Masson staining reagents to detect the deposition of collagen and stained with Sirius red staining reagents to measure the type and content of collagen. The pathological observation was evaluated by a Nikon microscope with a digital camera and polarization microscope.

\section{Western immunoblotting}

Western immunoblotting analysis of liver tissue was undertaken using the following monoclonal antibodies: $\beta$ actin was purchased from Zsbio (Beijing, China). a-SMA was purchased from Abcam Inc. (Shanghai, China). JAK2, phosphor-JAK2 (or p-JAK2), phosphor-ErK (or p-ErK), Erk, Akt, phosphor-Akt (or p-Akt) and cleaved Caspase 3 were purchased from CST (Beverly, MA). Rock1, P-53, Bcl2, and Bax were purchased from Santa Cruz biotechnology (Santa Cruz, CA). These antibodies were used to identify proteins expressed. The extracted proteins were subjected to electrophoresis on a $10 \%$ SDS-PAGE after normalization for protein content. After resolution, the protein samples were electrotransferred onto PVDF.

The membrane was blocked for $1 \mathrm{~h}$ in $0.1 \%$ nonfat dry milk in PBS. Membranes were incubated overnight at room temperature with the primary antibody in TBS. Membranes were washed twice for $5 \mathrm{~min}$ in PBS before the addition of the secondary antibody in TBS containing $0.1 \%$ nonfat dry 
milk for $1 \mathrm{~h}$. The membranes were then washed in TBS for $5 \mathrm{~min}$ followed by water for $5 \mathrm{~min}$. Reactive bands were identified using enhanced chemiluminescence and autoradiography according to the manufacturer's instructions.

\section{Statistical analysis}

Quantitative data was demonstrated as the mean \pm standard deviation (SD). The significance of the difference $v s$. the $\mathrm{CCl}_{4}$ group was analyzed by a Student's $t$-test. A MannWhitney rank sum test was used to measure the degree of histopathological liver fibrosis. The $P$-value less than 5\% $(P<0.05)$ was considered to be statistically significant.

\section{Results}

The effect of baicalin on body weight, liver, spleen, and kidney index The roles of baicalin on the rats' body weight, and the index of liver, spleen and kidney were evaluated, as shown in Fig. 1. The liver, kidney and spleen index were measured as the percentage of the total body weight. The results in Figs. 1B and $1 \mathrm{C}$ show that the body weight, the liver, kidney, and spleen index do not exhibit any statistically difference between normal and liver-fibrosis rats. The baicalin treatment does not have a significant impact on these factors.

\section{The effects of baicalin on liver function}

The effect of baicalin on the liver function was evaluated by measuring the therapeutic serum regimens, hyaluronic acid
(HA), Procollagen type III (HPCIII), Aspartate aminotransferase (ALT), and Alanine aminotransferase (AST), on the hepatic fibrosis in $\mathrm{CCl}_{4}$ rat model (Hu et al., 2010; Ozer et al., 2010). The results are summarized in Fig. 2. The significantly higher levels of HA, HPCIII, AST and ALT in the rats' serum in the $\mathrm{CCl}_{4}$ group were observed than that of the control group. However, HA and HPCIII levels (Figs. 2A and 2B) were effectively reduced at a higher dose of baicalin (above $100 \mathrm{mg} / \mathrm{kg}$ ). For example, by treating with $400 \mathrm{mg} / \mathrm{kg}$ baicalin, the HA and HPCII levels were reduced significantly by approximately $80 \%$ and $40 \%$, respectively. Additionally, baicalin also significantly suppressed $\mathrm{CCl}_{4}$-induced increase in AST level at relatively lower baicalin dose (Fig. 2D), while ALT level does not show a notably change upon treating baicalin (Fig. 2C). This result indicated that baicalin has a protective effect for the $\mathrm{CCl}_{4}$-induced liver injuries.

\section{The baicalin effect of hepatic activities}

Next, we investigated the effect of baicalin on the hepatic antifibrotic and anti-oxygenation capability by evaluating the hydroxyproline (HyP), malondialdehyde (MDA), glutathione peroxidase (GSH-Px), and superoxide dismutase (SOD) in liver. $\mathrm{HyP}$ is an amino acid, and it exists almost exclusively in collagens (Alcock et al., 2019; Katayama et al., 1997; Kim et al., 2009). Measurement of HyP in fibrotic tissues has been used as a reliable method to study fibrosis and to investigate the potentially anti-fibrotic agent (Kim et al., 2009). As shown in Fig. 3A, the
(A)

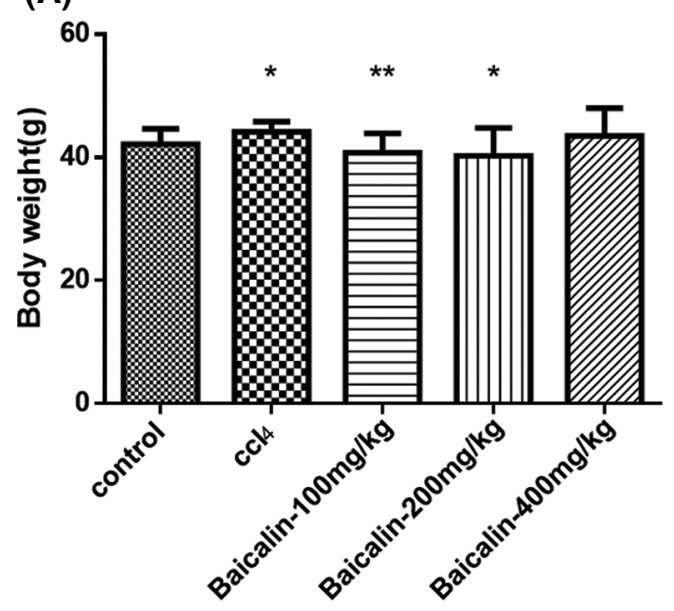

(C)

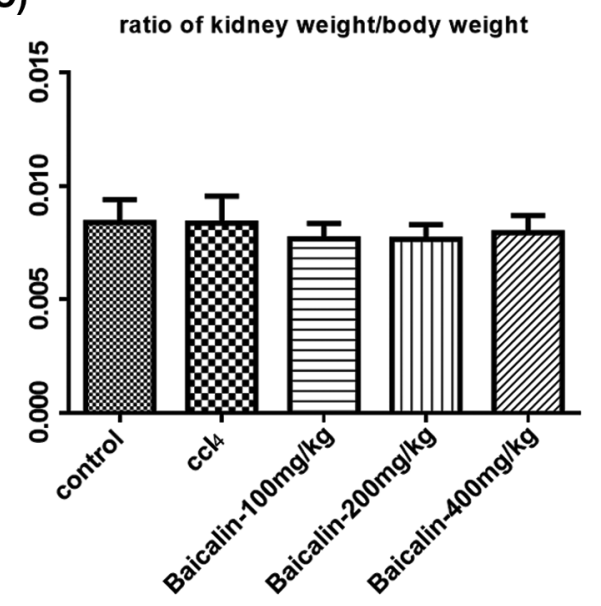

(B)

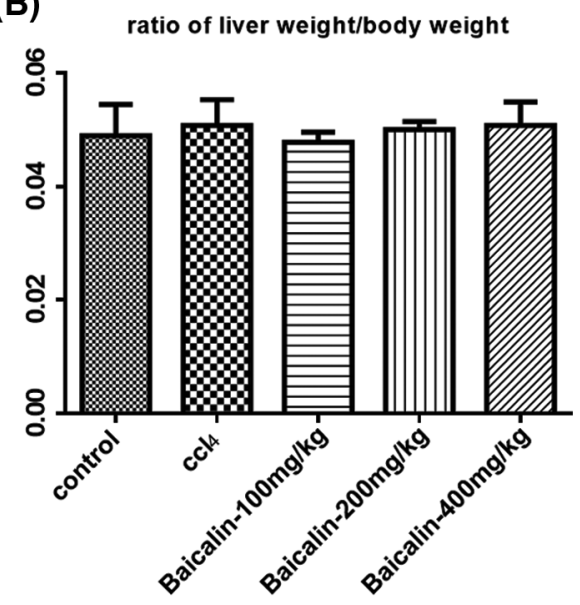

(D)

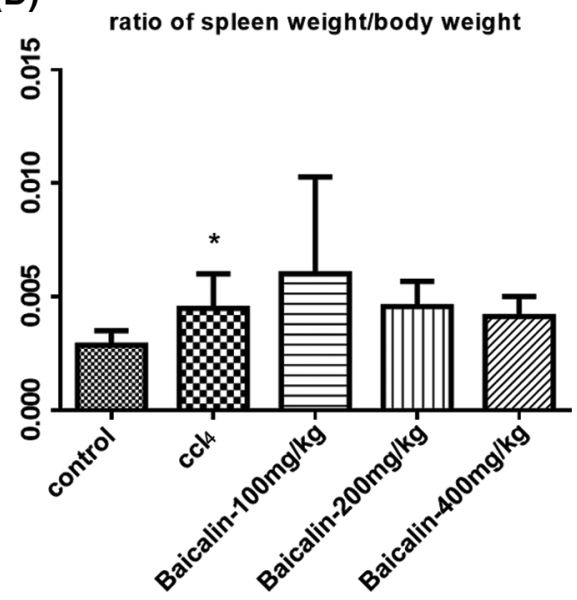

FIGURE 1. Body weight (A), liver (B), kidney $(\mathrm{C})$ and spleen (D) index in the different groups.

Data were presented as mean \pm SD. ${ }^{*}$ and ${ }^{\star *}$ represent the significant difference from the control group with $P<0.5$, and $P<$ 0.01 , respectively. 
(A)

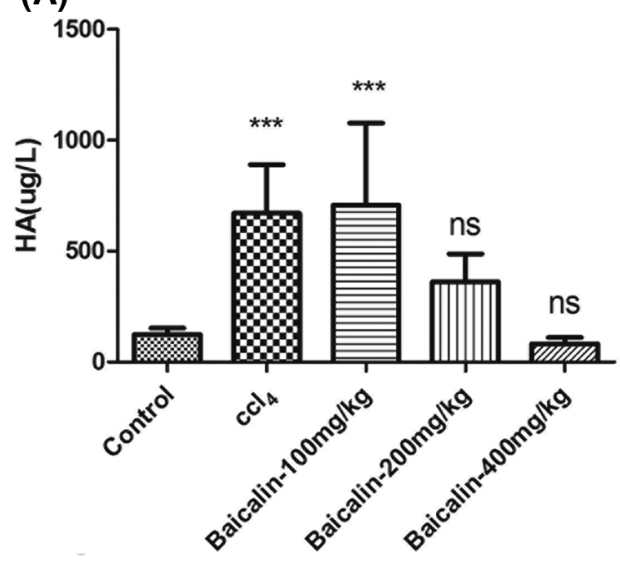

(C)

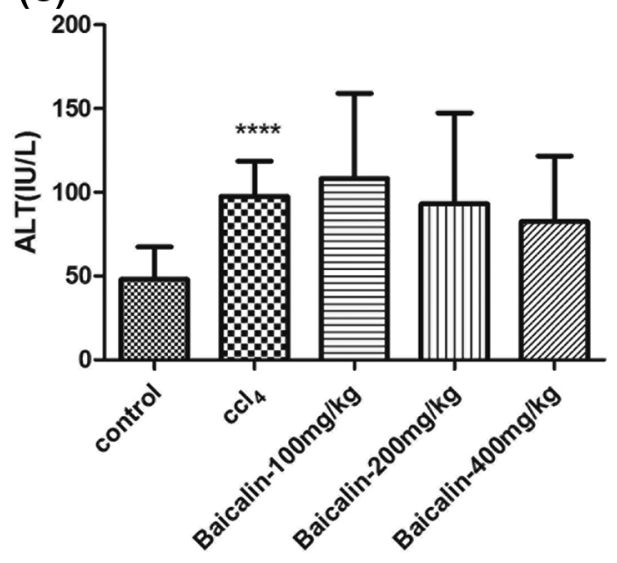

(B)

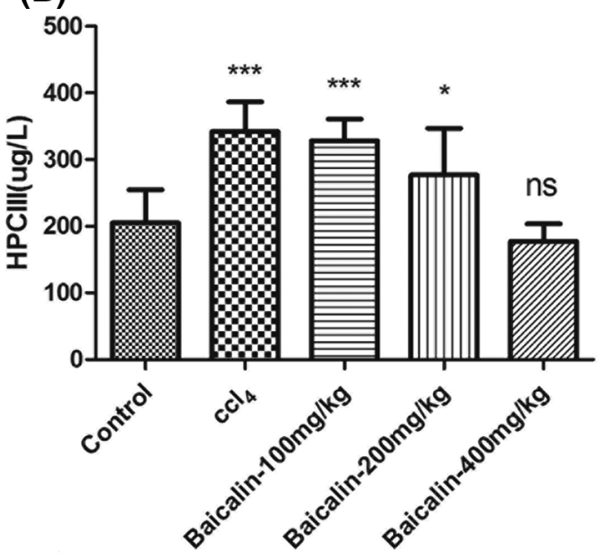

(D)

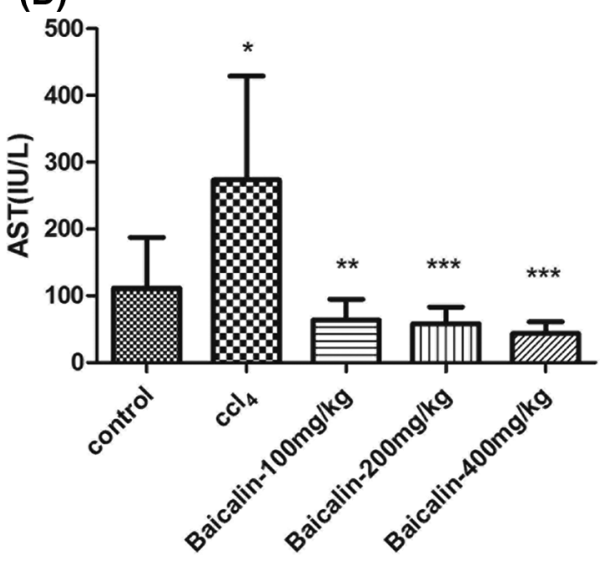

(B)

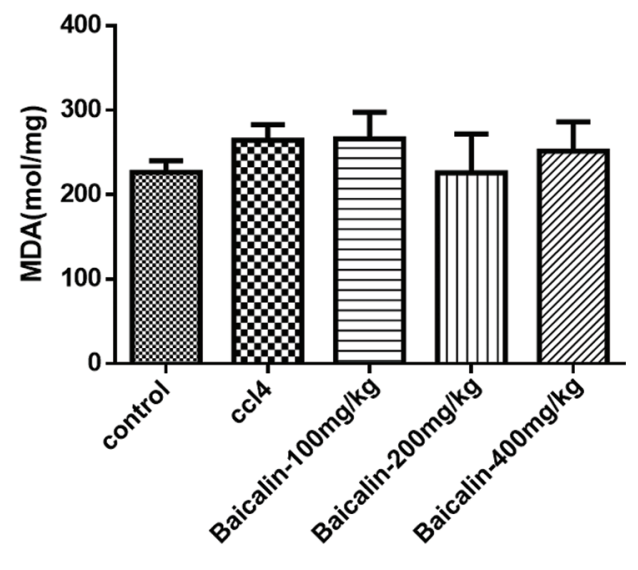

(D)

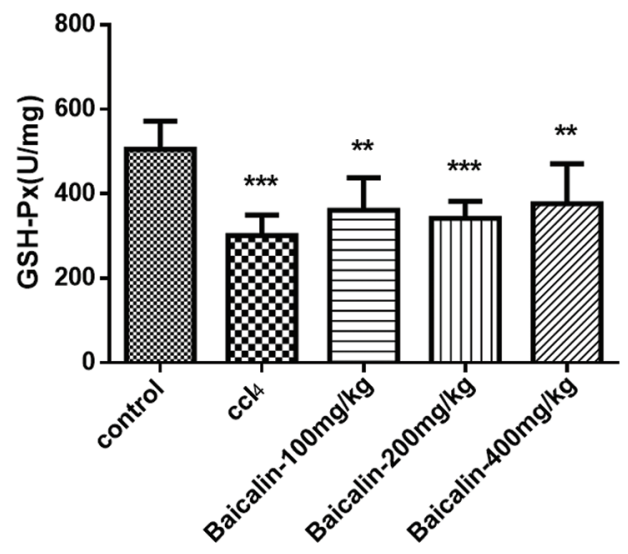

FIGURE 2. Hyaluronic acid (HA, A), Procollagen type III (HPCIII, B), Aspartate aminotransferase (ALT, C) and Alanine aminotransferase (AST, D) in different experimental groups. Data were expressed as mean \pm SD. * ${ }^{* *},{ }^{* * *}$, and ${ }^{* * *}$ represent the significant difference from the control group with $P<0.5, P<0.01, P<$ 0.001 , and $P<0.0001$, respectively.
(A)

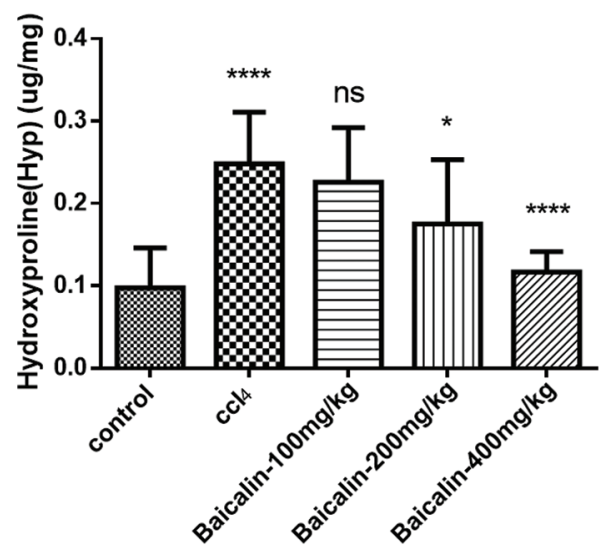

(C)

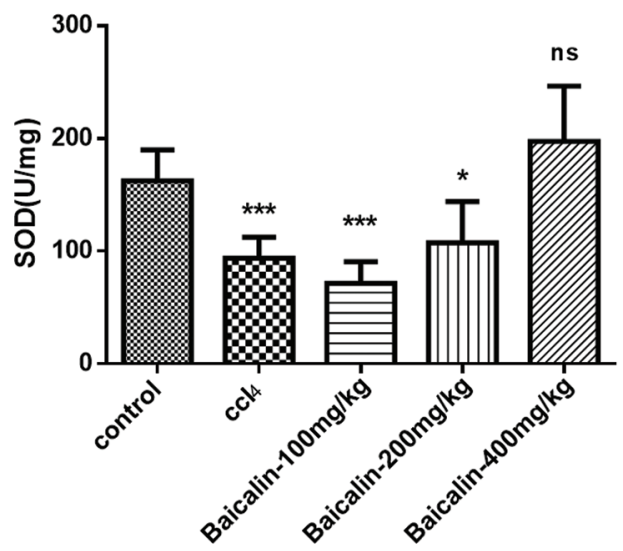


content of $\mathrm{HyP}$ in the livers after treating $\mathrm{CCl}_{4}$ were significantly higher than those in the normal livers, suggesting the liver fibrosis has been formed in the $\mathrm{CCl}_{4}$ treated rats. After treating different doses of baicalin, the hepatic HyP levels were markedly decreased. This finding suggests that baicalin is an effective anti-fibrotic agent in $\mathrm{CCl}_{4}$ injury rats.

Moreover, MDA, SOD, and GSH-Px are commonly used as biomarkers to evaluate the oxidative stress and the antioxidant status in liver injuries (Katayama et al., 1997; Romani et al., 1988; Sampey et al., 2003). As can be seen in Figs. 3C and 3D, GSH-Px and SOD levels are reduced in $\mathrm{CCl}_{4}$-groups, suggesting that the end-product of lipid peroxidation due to the oxygenation process is higher in $\mathrm{CCl}_{4}$ group than in the control group. As increasing the dose of baicalin treatment (Figs. 3C and 3D), the SOD and GSH-Px levels effectively increase. In contract, MDA level does not display a statistical difference among the group studied (Fig. 3B).

The effect of protein expression level in the liver tissue Liver fibrosis is often investigated by the accumulation of proteins in extracellular matrix, which occurs in the most types of chronic liver diseases (Chen et al., 2015; Li et al., 2019). To determine whether the protective effect of baicalin on $\mathrm{CCl}_{4}$-induced liver injury is mediated by cell apoptosis, the protein levels of AKT, phosphorylate (p-) AKT, ERK, and phosphorylate (p-) ERK in cell were detected. The results indicated that $\mathrm{CCl}_{4}$ injection enhanced the levels of AKT, p-AKT, p-JAK2, ERK, p-ERK and ROCK1, compared with the levels in control group (Figs. 4 and 5). However, as shown in Fig. 5, treating with a small dose of baicalin does not significantly affect the p-JAK2 and ERK level in $\mathrm{CCl}_{4^{-}}$ injuried rats. These levels were only suppressed significantly

\section{A B C D E}

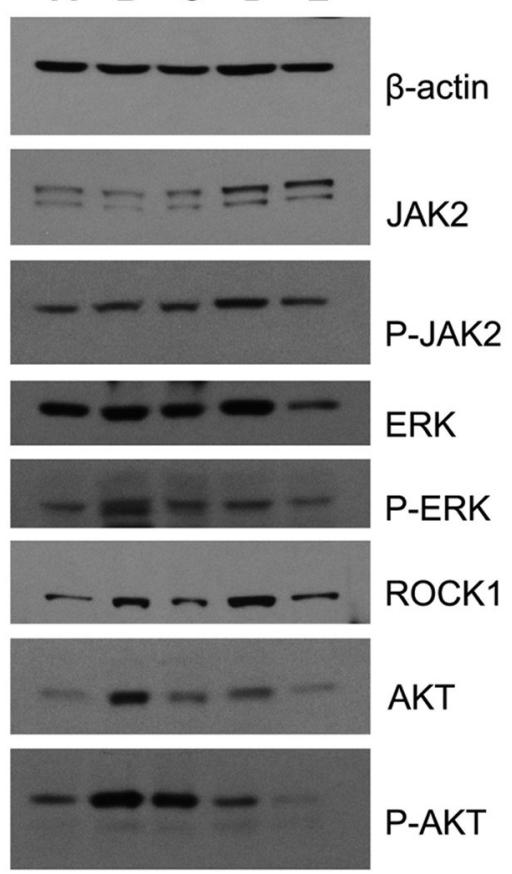

FIGURE 4. The effect of baicalin on $\beta$-actin, JAK2, p-ERK, ROCK1, $\mathrm{AKT}$, and $\mathrm{P}-\mathrm{AKT}$ in the rats' livers.

(A) Control group, (B) $\mathrm{CCl}_{4}$ control group, (C) baicalin (100 mg/kg),

(D) baicalin $(200 \mathrm{mg} / \mathrm{kg})$, (E) baicalin $(400 \mathrm{mg} / \mathrm{kg})$.

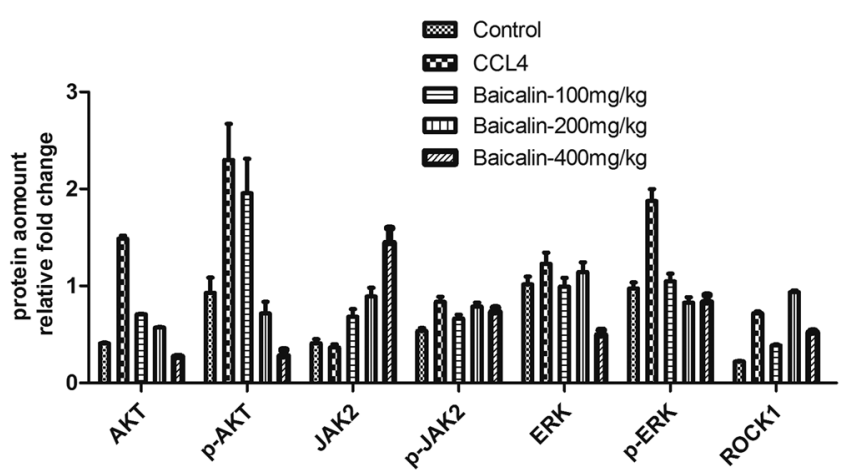

FIGURE 5. The effect of baicalin on AKT/ JAK2/ ERK and ROCK protein expression in the rats' livers.

at higher doses of baicalin. On the other hand, a small dose of baicalin $(100 \mathrm{mg} / \mathrm{kg})$ can significantly suppress AKT, P$\mathrm{AKT}$, and ROCK1 level in the $\mathrm{CCl}_{4}$-injuried rats. It has been shown that high responsiveness of cell apoptosis is related to the increased expression of AKT and ROCK1 levels in liver cells (Liu et al., 2015b). Our findings indicate that baicalin treatment may suppress the AKT and ROCK1 signaling pathway in $\mathrm{CCl}_{4}$ induced liver injury, suggesting the potential capability of baicalin to suppress cell apoptosis.

B-cell lymphoma-2 (Bcl-2) is considered to be an antiapoptotic protein, and Bcl-2 associated $\mathrm{X}$ protein (Bax), mammalian target of rapamycin (mTOR), p53 and caspase3 are known to be the biomarker of apoptosis (Cao et al., 2013; Guo et al., 2002). To better understand the role of baicalin on the cell apoptosis in liver injury, the western blot analysis was performed to detect the expression of apoptosis markers at mRNA and protein levels. As indicated in Figs. 6 and 7 , the relative expression of $\mathrm{Bax}, \mathrm{Bcl}-2, \mathrm{mTOR}, \mathrm{p} 53$, and

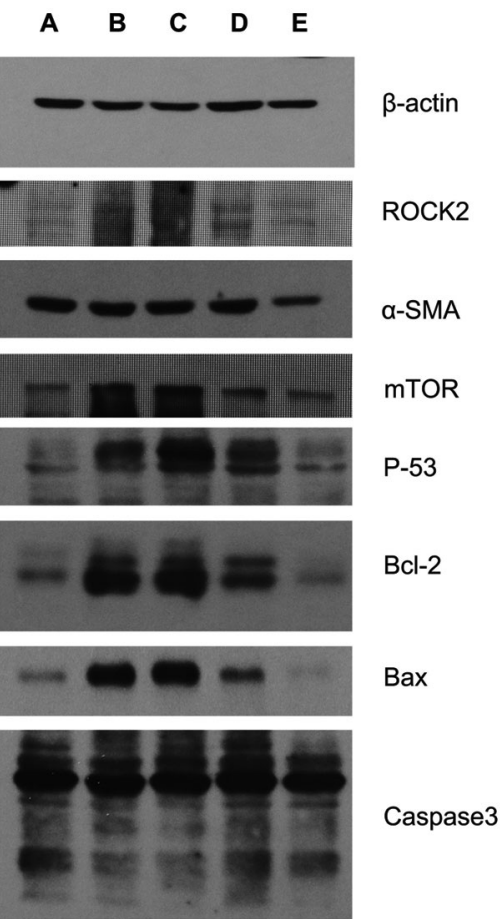

FIGURE 6. The effect of baicalin on $\beta$-actin, ROCK2, $\alpha$-SMA, mTOR, p-53, Bcl-2, Bax in the rats' livers mediated by cell apoptosis. (A) Control group, (B) $\mathrm{CCl}_{4}$ control group, (C) baicalin $(100 \mathrm{mg} / \mathrm{kg}$ ), (D) baicalin $(200 \mathrm{mg} / \mathrm{kg})$, (E) baicalin $(400 \mathrm{mg} / \mathrm{kg})$. 


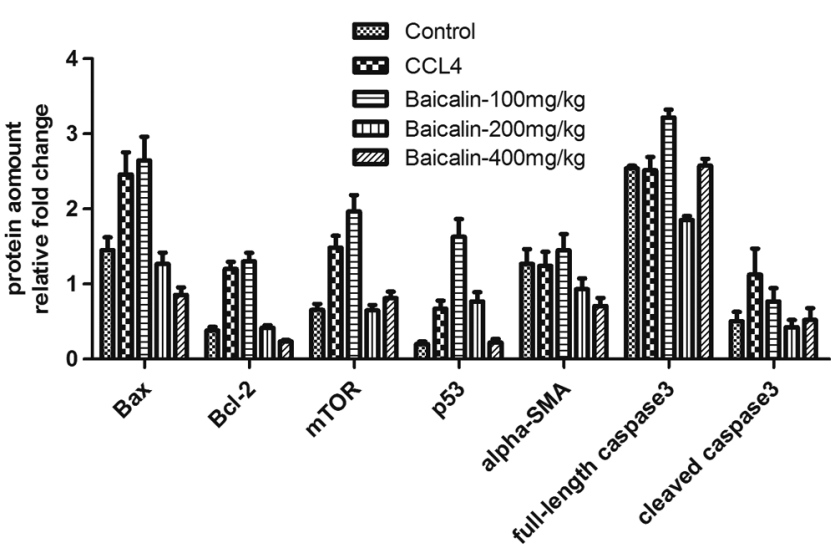

FIGURE 7. The effect of baicalin on the cell apoptosis in the rats' livers.

cleaved caspase-3 was significantly enhanced after $\mathrm{CCl}_{4}$ treatment, compared with the control. However, this enhancement was markedly attenuated by treatment of baicalin. Several studies have shown that high level of Bax, Bcl-2, mTOR, and p53 associates with cell apoptosis (Gao et al., 2002, 2003; Liu et al., 2019). Thus, these results suggest that baicalin may attenuate hepatocyte apoptosis in liver injury in the dose dependence matter.

\section{Histological staining analysis}

Histological characteristics of the liver in the control group showed a normal lobular architecture and structure (Fig. 8A). However, after treating with $\mathrm{CCl}_{4}$, the extensive hepatocellular damages were observed, as evidenced by the presence of hepatocellular degeneration and infiltration inflammatory cells (Fig. 8B). The treatment of baicalin in various dosages can attenuate the degree of injury changes (Figs. 8C-8E). These results confirmed that baicalin has the anti-fibrotic effects in $\mathrm{CCl}_{4}$-liver injury model, and the effect is dose dependent.

The effect of baicalin on the changes of liver cells was presented in Fig. 9. The VG staining results indicated that $\mathrm{CCl}_{4}$ treated liver showed extensive changes in microstructures, including marked enlarged domains of portal inflammation, hepatic necrosis, and fibrotic septa (Fig. 9B). No abnormalities were observed in the control group (Fig. 9A). Baicalin treatment could significantly alleviate the degree of liver injury in the cellular level, and their cell morphologies are close to that of normal cells.

\section{Discussion}

Herein, we evaluate the role of baicalin on liver fibrosis induced by $\mathrm{CCl}_{4}$ in rats. The treatment of $\mathrm{CCl}_{4}$ in rats has been commonly used as an ideal model system to study liver fibrosis (Kodavanti et al., 1989; Ritesh et al., 2015). In this work, liver fibrosis was induced successfully by subcutaneous $2 \% \mathrm{CCl}_{4}$ injection for 6 weeks. The histological data has showed that the microstructure of liver lobules was destroyed and pseudolobules generated. In addition, the high hydroxyproline level in liver, as well as high HA, HPCIII, and AST in serum consistently confirmed the formation of liver fibrosis.

We showed that baicalin treatment appeared to be beneficial in this model of $\mathrm{CCl}_{4}$ injury by means of improving liver function, reducing liver index, and inhibiting the process of developed hepatic fibrosis. This protective function could associate with the increase of SOD and GSHPx activities and the reduction of MDS level, thus suggesting antioxidant activity may be the possible mechanism of baicalin in liver fibrosis. Additionally, baicalin can suppress several biomarkers associated with cell apoptosis in $\mathrm{CCl}_{4}$ induced liver cells. It indicates that baicalin can alleviate liver cell injury by suppressing the cell apoptosis process.

The increased content of aminotransferase (AST) could be due to the leakage from the damaged hepatic cells and has been used as a marker for liver injury (Hu et al., 2010; Ozer et al., 2010). It was shown in Fig. 2D that the abnormally high AST level was noted in rats treated with $\mathrm{CCl}_{4}$. AST level is reduced by administration of baicalin. This finding provided strong evidence that the baicalin can improve the hepatic damages in rats without significant hepatotoxicity. The role of baicalin was further evaluated by the improvement in the histopathological analysis.
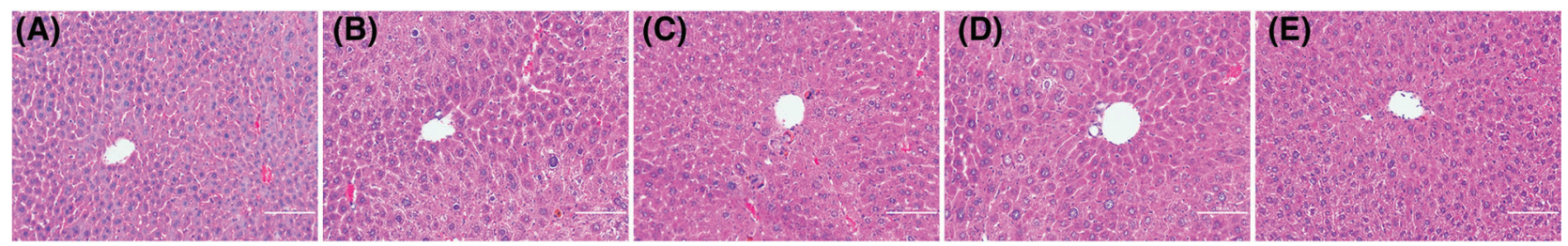

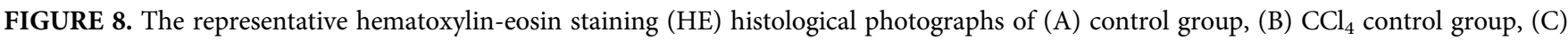
$\mathrm{CCl}_{4}$ and low-dose baicalin group (100 mg/kg), (D) $\mathrm{CCl}_{4}$ and intermediate-dose baicalin group (200 mg/kg), (E) CCl ${ }_{4}$ and high-dose baicalin group $(400 \mathrm{mg} / \mathrm{kg})$.
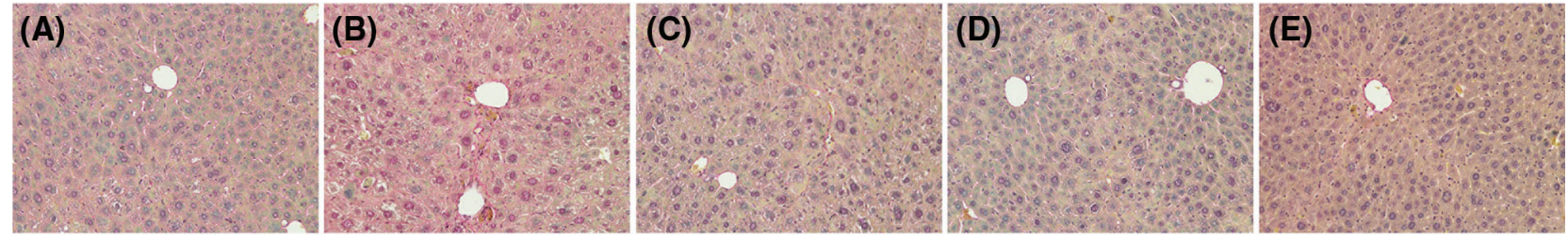

FIGURE 9. Representative Van Gieson's (VG) Stain photographs $x 200$ of (A) control group, (B) $\mathrm{CCl}_{4}$ control group, (C) CCl ${ }_{4}$ and low-dose baicalin group (100 mg/kg), (D) $\mathrm{CCl}_{4}$ and intermediate-dose baicalin group (200 mg/kg), (E) $\mathrm{CCl}_{4}$ and high-dose baicalin group (400 mg/kg). 
HPCIII and HA levels in serum are key markers of hepatic fibrogenesis. Hepatic HA level reflects the total amount of collagen, and it has been used to evaluate the extent of fibrosis (Alcock et al., 2019; Kim et al., 2009). Therefore, HPCIII and HA are important indexes to evaluate liver fibrosis. The result shown in Fig. 2 demonstrated that baicalin could significantly reduce HA and PCIII in serum, which suggested that baicalin could suppress deposition and accumulation of collagen in the liver.

\section{Conclusion}

The present study showed that baicalin from traditional medicine herb, Scutellaria baicalensis, has a rich bioactive profile and has preventive functions to liver fibrosis. The possible mechanism of the effects might be related to its antioxidant activities and suppression of cell apoptosis, decreasing the level of Hyp, SOD, GSH-Px, AKT/JAK2/ERK protein and inhibition of collagen synthesis. Baicalin could be a potential agent for liver fibrosis treatment, which targeting the apoptosis of hepatocytes and preventing cell oxidation. This research can provide alternative options for the development of pharmaceutical therapeutic compounds in medical practices.

Availability of Data and Materials: The datasets generated during and/or analysed during the current study are available from the corresponding author on reasonable request.

Author Contribution: The authors confirm contribution to the paper as follows: study conception and design: MengTing Li; data collection: Kaifeng Zheng; analysis and interpretation of results: Yi-er Qiu; draft manuscript preparation: Mengting Li. All authors reviewed the results and approved the final version of the manuscript.

Ethics Approval: The animal study was approved by the Institutional Animal Care and Use Committee of Shanghai Rat \& Mouse Biotech Co., Ltd. (SHRM) (Approval No. SHRM-IACUC-045). It was carried out throughout the experiment following the international laboratory animal use and care guideline.

Funding Statement: The authors received no specific funding for this study.

Conflicts of Interest: The authors declare that they have no conflicts of interest to report regarding the present study.

\section{References}

Alcock RD, Shaw GC, Tee N, Welvaert M, Burke LM (2019). Urinary hydroxyproline is only suitable as a biomarker for acute intake, up to $6 \mathrm{hr}$ postingestion of collagen proteins in "free-living," healthy, active males. International Journal of Sport Nutrition and Exercise Metabolism 29: 461-465.

Are VS, Vuppalanchi R, Vilar-Gomez E, Chalasani N (2020). Enhanced liver fibrosis score can be used to predict liverrelated events in patients with nonalcoholic steatohepatitis and compensated cirrhosis. Clinical Gastroenterology and Hepatology 19: 1292-1293.

Cao J, Chen J, Wang J, Jia R, Xue W et al. (2013). Effects of fluoride on liver apoptosis and Bcl-2, Bax protein expression in freshwater teleost, Cyprinus carpio. Chemosphere 91: 1203-1212.

Cederbaum AI, Lu Y, Wu D (2009). Role of oxidative stress in alcoholinduced liver injury. Archives of Toxicology 83: 519-548.

Chen W, Han C, Zhang J, Song K, Wang Y et al. (2015). miR-150 deficiency protects against FAS-induced acute liver injury in mice through regulation of AKT. PLoS One 10: e0132734.

Dai LJ, Li HY, Guan LX, Ritchie G, Zhou JX (2009). The therapeutic potential of bone marrow-derived mesenchymal stem cells on hepatic cirrhosis. Stem Cell Research 2: 16-25.

Dong Y, Xing Y, Sun J, Sun W, Xu Y, Quan C (2020). Baicalein alleviates liver oxidative stress and apoptosis induced by high-level glucose through the activation of the PERK/Nrf2 signaling pathway. Molecules 25: 599.

Friedman SL (2003). Liver fibrosis-from bench to bedside. Journal of Hepatology 38: S38-S53.

Gao D, Sakurai K, Chen J, Ogiso T (1995). Protection by baicalein against ascorbic acid-induced lipid peroxidation of rat liver microsomes. Research Communications in Molecular Pathology and Pharmacology 90: 103-114.

Gao CJ, Sun WY, Christofidou-Solomidou M, Sawada M, Newman DK et al. (2003). PECAM-1 functions as a specific and potent inhibitor of mitochondrial-dependent apoptosis. Blood 102: 169-179.

Gao XS, Zhang Y, Arrazola P, Hino O, Kobayashi T et al. (2002). Tsc tumour suppressor proteins antagonize amino-acid-TOR signalling. Nature Cell Biology 4: 699-704.

Gebhardt R (2002). Oxidative stress, plant-derived antioxidants and liver fibrosis. Planta Medica 68: 289-296.

Guo XZ, Shao XD, Liu MP, Xu JH, Ren LN et al. (2002). Effect of bax, bcl-2 and bcl-xL on regulating apoptosis in tissues of normal liver and hepatocellular carcinoma. World Journal of Gastroenterology 8: 1059-1062.

$\mathrm{Hu}$ KQ, Schiff ER, Kowdley KV, Min AD, Shiffman ML et al. (2010). Histologic evidence of active liver injury in chronic hepatitis $B$ patients with normal range or minimally elevated alanine aminotransferase levels. Journal of Clinical Gastroenterology 44: $510-516$.

Huang HL, Wang YJ, Zhang QY, Liu B, Wang FY et al. (2012). Hepatoprotective effects of baicalein against $\mathrm{CCl}(4)$ induced acute liver injury in mice. World Journal of Gastroenterology 18: 6605-6613.

Huang X, Mao W, Zhang T, Wang M, Wang X et al. (2018). Baicalin promotes apoptosis and inhibits proliferation and migration of hypoxia-induced pulmonary artery smooth muscle cells by upregulating $\mathrm{A} 2 \mathrm{a}$ receptor via the SDF-1/CXCR4 signaling pathway. BMC Complementary and Alternative Medicine 18: 330.

Huang X, Wang X, Lv Y, Xu L, Lin J et al. (2014). Protection effect of kallistatin on carbon tetrachloride-induced liver fibrosis in rats via antioxidative stress. PLoS One 9: e88498.

Katayama T, Yokoyama S, Mitomi T, Watanabe K (1997). Alterations in glutathione peroxidase activity following reperfusion injury to rat liver. Tokai Journal of Experimental and Clinical Medicine 22: 33-44.

Kim KY, Cho SE, Yu BS (2009). Intragastrically applicated CCl4thiopental sodium enhanced lipid peroxidation and liver fibrosis (cirrhosis) in rat: malonedialdehyde as a parameter of lipid peroxidation correlated with hydroxyproline as a parameter of collagen synthesis (deposition). Toxicological Research 25: 71-78.

Kisseleva T, Brenner DA (2006). Hepatic stellate cells and the reversal of fibrosis. Journal of Gastroenterology and Hepatology 21: S84-S87. 
Kodavanti PR, Joshi UM, Young RA, Meydrech EF, Mehendale HM (1989). Protection of hepatotoxic and lethal effects of CCl4 by partial hepatectomy. Toxicologic Pathology 17: 494-505.

Li S, Yi Z, Deng M, Scott MJ, Yang C et al. (2019). TSLP protects against liver $\mathrm{I} / \mathrm{R}$ injury via activation of the PI3K/Akt pathway. JCI Insight 4: e129013.

Lin L, Zhou F, Shen S, Zhang T (2018). Fighting liver fibrosis with naturally occurring antioxidants. Planta Medica 84: 13181333.

Liu A, Huang L, Fan H, Fang H, Yang Y et al. (2015a). Baicalein pretreatment protects against liver ischemia/reperfusion injury via inhibition of $\mathrm{NF}-\kappa \mathrm{B}$ pathway in mice. International Immunopharmacology 24: 72-79.

Liu A, Wang W, Fang H, Yang Y, Jiang X et al. (2015b). Baicalein protects against polymicrobial sepsis-induced liver injury via inhibition of inflammation and apoptosis in mice. European Journal of Pharmacology 748: 45-53.

Liu Y, Jia L, Min D, Xu Y, Zhu J, Sun Z (2019). Baicalin inhibits proliferation and promotes apoptosis of vascular smooth muscle cells by regulating the MEG3/p53 pathway following treatment with oxLDL. International Journal of Molecular Medicine 43: 901-913.

Mccay PB, Lai EK, Poyer JL, Dubose CM, Janzen EG (1984). Oxygenand carbon-centered free radical formation during carbon tetrachloride metabolism. Observation of lipid radicals in vivo and in vitro. Journal of Biological Chemistry 259: 2135-2143.

Ozer JS, Chetty R, Kenna G, Palandra J, Zhang Y et al. (2010). Enhancing the utility of alanine aminotransferase as a reference standard biomarker for drug-induced liver injury. Regulatory Toxicology and Pharmacology 56: 237-246.

Poli G (2000). Pathogenesis of liver fibrosis: Role of oxidative stress. Molecular Aspects of Medicine 21: 49-98.

Ritesh KR, Suganya A, Dileepkumar HV, Rajashekar Y, Shivanandappa $\mathrm{T}$ (2015). A single acute hepatotoxic dose of CCl4 causes oxidative stress in the rat brain. Toxicology Reports 2: 891-895.

Romani F, Vertemati M, Frangi M, Aseni P, Monti R et al. (1988). Effect of superoxide dismutase on liver ischemiareperfusion injury in the rat: A biochemical monitoring. European Surgical Research 20: 335-340.
Sampey BP, Korourian S, Ronis MJ, Badger TM, Petersen DR (2003). Immunohistochemical characterization of hepatic malondialdehyde and 4-hydroxynonenal modified proteins during early stages of ethanol-induced liver injury. Alcoholism: Clinical and Experimental Research 27: 1015-1022.

Sastre J, Serviddio G, Pereda J, Minana JB, Arduini A et al. (2007). Mitochondrial function in liver disease. Frontiers in Bioscience 12: 1200-1209.

Sun WY, Wang L, Liu H, Li X, Wei W (2012). A standardized extract from Paeonia lactiflora and Astragalus membranaceus attenuates liver fibrosis induced by porcine serum in rats. International Journal of Molecular Medicine 29: 491-498.

Sun WY, Wei W, Wu L, Gui SY, Wang H (2007). Effects and mechanisms of extract from Paeonia lactiflora and Astragalus membranaceus on liver fibrosis induced by carbon tetrachloride in rats. Journal of Ethnopharmacology 112: $514-523$.

Toledano MB, Mukherjee SK, Howell J, Westaby D, Khan SA et al. (2019). The emerging burden of liver disease in cystic fibrosis patients: A UK nationwide study. PLoS One 14: e0212779.

Wu R, Murali R, Kabe Y, French SW, Chiang YM et al. (2018). Baicalein targets GTPase-mediated autophagy to eliminate liver tumor-initiating stem cell-like cells resistant to mTORC1 inhibition. Hepatology 68: 1726-1740.

Yin H, Huang L, Ouyang T, Chen L (2018). Baicalein improves liver inflammation in diabetic $\mathrm{db} / \mathrm{db}$ mice by regulating HMGB1/TLR4/NF- $\kappa B$ signaling pathway. International Immunopharmacology 55: 55-62.

Yu Z, Li Q, Wang Y, Li P (2020). A potent protective effect of baicalein on liver injury by regulating mitochondria-related apoptosis. Apoptosis 25: 412-425.

Zhou HC, Wang H, Shi K, Li JM, Zong Y, Du R (2018). Hepatoprotective effect of baicalein against acetaminopheninduced acute liver injury in mice. Molecules 24: 131.

Zimmermann A, Darstein F, Hoppe-Lotichius M, Toenges G, Lautem A et al. (2019). Cirrhosis risk score of the donor organ predicts early fibrosis progression after liver transplantation. Journal of Gastrointestinal and Liver Diseases 28: 53-61. 